\title{
Estimation of RF PA Nonlinearities after Cross-correlating Current and Output Voltage
}

\author{
Pedro Fonseca Mota • José A. Machado da Silva • \\ Ricardo A. Veiga
}

Received: 9 September 2009 /Accepted: 16 November 2009/Published online: 12 January 2010

(C) Springer Science+Business Media, LLC 2010

\begin{abstract}
The estimation of $1 \mathrm{~dB}$ compression and thirdorder intercept points can be obtained after the crosscorrelation between dynamic current and output voltage of radio frequency power amplifiers. This estimation is performed using actual power measurements and not power inferred from voltage values. The underlining theory and a correlator that allows implementing this measurement onchip are presented. The trade-off between measuring voltage and the actual power is also discussed and it is shown that different information concerning the output load is obtained when observing the PA's output voltage and power. Simulation results, obtained with the model of a prototype demonstration chip, show that good accuracy can be obtained with relatively simple measurement conditions. These results include the analysis of optimum stimuli amplitudes and the effect of noise in estimation accuracy.
\end{abstract}

Keywords RF test - Cross-correlation - RF power-amplifier Non-linearity

Responsible Editor: K. Arabi

P. F. Mota $(\varangle) \cdot J$. A. Machado da Silva $\cdot$ R. A. Veiga INESC Porto, Faculdade de Engenharia, Universidade do Porto, Rua Dr. Roberto Frias, Campus FEUP,

Porto 4200-465, Portugal

e-mail: dee05001@fe.up.pt

J. A. Machado da Silva

e-mail: jms@fe.up.pt

R. A. Veiga

e-mail: ee08064@fe.up.pt

\section{Introduction}

The development of on-chip RF testing methods has motivated different research activities in the past decade $[5,6,8,12,15,16]$. Nonlinearity estimation is among the most important test operations which have driven this research [7, 9]. That is, e. g., the case with circuits operating with complex modulations such as Widebandwidth Code-Division for Multiple Access (W-CDMA) and CDMA2000, which present a high degree of amplitude variation or, in other words, a high crest factor (CF) instantaneous peak to carrier's rms voltage ratios of 10 to 30 can occur depending on formats and filtering. Furthermore, besides high peak-to-average ratios, these also change with base station call loading, large operating temperature ranges, and large transmit power ranges. High linearity levels are required for transceivers operating in these conditions to prevent signal compression or clipping. If the peaks of a modulated signal are clipped the emitter can fail its spectral mask requirements and a loss of data can occur.

Traditional RF testing relying on high-end laboratory instruments provide accurate measurements but is time consuming and expensive. On the other hand, accessing deeply embedded circuits requires delicate electronic probes. This is particularly true within modern system-on-chip (SoC) devices which incorporate different circuits and functionalities on a monolithic substrate, such as, high-speed digital, memory, analog, and RF circuits. This high scale integration of components makes SoC testing complex and costly [14]. Today's RF products are expected to not only provide excellent performance characteristics, but also to sustain them for several years of performance in the field, what brings a new degree of importance to embedded test and calibration circuits. 
The lack of harmonized and universal RF structural tests have forced industries to resort to specifications' functional testing in order to ensure the required low defect ppm (ideally zero) quality level, eventually relying on both builtin and built-out tests $[4,14]$.

The methodologies presented in $[1,11]$ allow testing an RF power amplifier (PA) for nonlinearity using a polynomial fitting approach, after applying single tone input stimuli sweeping the PA's dynamic range and on capturing the respective output voltage values. From the resulting set of pairs of values one can obtain the polynomial that best fits the PA's transfer characteristic and whose coefficients allow one to calculate $1 \mathrm{~dB}$ compression $\left(\operatorname{Pin}_{1 d B}\right)$ and third order intercept $\left(\operatorname{Pin}_{I I P 3}\right)$ points values.

By rectifying and averaging the RF output voltage, a measure of the power delivered by the PA can be obtained, having in mind that the load resistance is known. However, observing voltage (or current) only represents power in an indirect way. E. g., in case of a phase mismatch between voltage and current, RF power sensing based on observing one or the other might be insufficient. Furthermore, observing output power is important for controlling an RF PA, whose operation may be impaired by strong load fluctuations or mismatches. Large mismatches may lead to high current or voltage peaks that not only affect PA's linearity but may also shorten lifetime or lead to damage.

The methodology being presented here relies also on the polynomial fitting approach for estimating nonlinearities, however, measures are provided by the cross-correlation between output voltage and current, instead.

The remaining of the paper presents in Section 2 a comparison between observing output voltage and power for testing purposes. Section 3 describes the procedure being proposed to estimate $\operatorname{Pin}_{1 d B}$ and $\operatorname{Pin}_{I I P 3}$ from the power transfer characteristic curve, together with validating simulation results. Section 4 shows how power measurements can be obtained from the cross-correlation between voltage and current signals and Section 5 presents the mixer based circuit being used as a correlator. Section 6 describes preliminary simulation results obtained with a demonstration prototype chip meanwhile sent for fabrication. Finally, Section 7 highlights the main conclusions.

\section{Power vs. Voltage Measurement}

It has been shown that it is possible to estimate RF PA's 1 $\mathrm{dB}$ compression $\left(\operatorname{Pin}_{1 d B}\right)$ and third order intercept $\left(\operatorname{Pin}_{I I P 3}\right)$ points values after sampling both input and output voltages $[1,3,11,15]$. With these gathered samples one can find the coefficients that define the polynomial that best fits the PA's transfer characteristic.
However, these samples have to be captured in regions which comprise both linear and compression behaviors and, when observing voltage, take care that asymmetries are detected in the waveform [11]. When that is the case both positive and negative peaks have to be considered (the average of the positive and negative peak voltages has also been used) using maximum and minimum detectors, in order to get a full and correct characterization. On the other hand, the objective is actually to estimate power from $P=V_{r m s}^{2} / R_{L}$, assuming that the load impedance is known.

Observing power directly does not provide information on asymmetries in the positive and negative peak values of the voltage waveforms, as those are either hidden by the power detector or filtered out if the detector only evaluates the power of the fundamental component. Unless one operates in the linear region and with well known waveforms, it is not possible to infer accurately about the voltage peak amplitudes. The presence of an nth harmonic with amplitude A causes a fractional error in the output voltage measurement of, in the worst case, $A$ (n odd) or $n^{2} A^{2} / 2$ (n even), and twice these values in the output power measurement [13].

When working at PA's full power, where compression and clipping are likely to occur, the amplitude of the signal to be amplified is smeared among harmonic components which may present significant power relatively to the fundamental, making the estimation of power from the fundamental peak voltage, no longer possible. If the bandwidth of the measuring detectors is narrow an additional error occurs due to the reduction of the signal amplitude in the measurement bandwidth.

Figure 1 shows the $i_{D S} / v_{D S}$ curves of the MOS transistor of a generic RF PA (not taking into consideration the Early

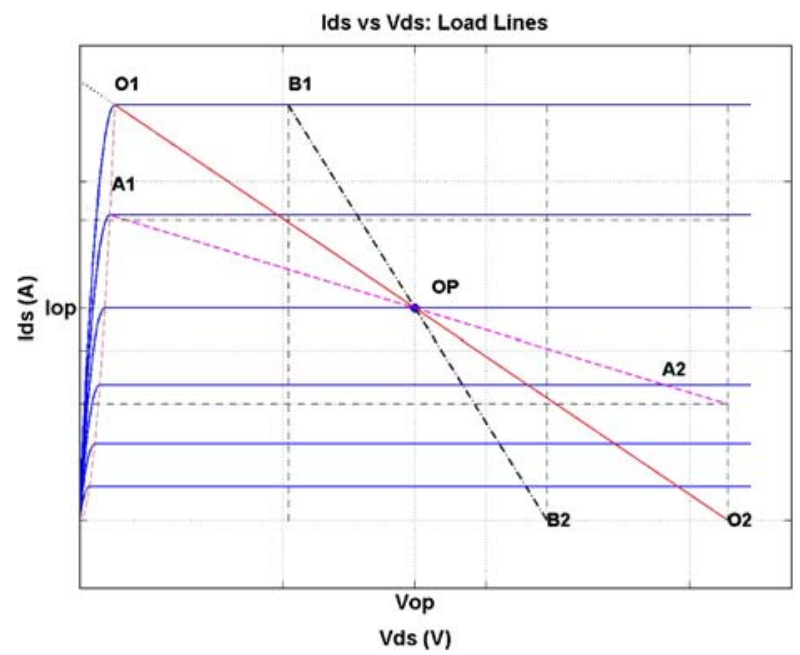

Fig. 1 Load lines different from the optimum one (line O1-O2) rend smaller output powers due to smaller $i_{D S}$ and $v_{D S}$ excursions 
effect) parameterized for different $v_{G S}$ values, superimposed with three different load lines.

When designing a PA, its load should be set to the optimum load value (Jacobi's law) that maximizes power transfer [10]. Considering a linear operation, the application of an input signal rends $\Delta v_{d s}=-\Delta i_{d} \cdot R_{L}$. In the $i_{D S} / v_{D S}$ curves shown in Fig. 1, the maximum power transfer occurs when voltage and current show their maximum excursions. This corresponds to the optimum load line (01-02) plotted in solid, which also defines the $i_{D S} v_{D S}$ triangle with the largest area. The triangles defined by the dash-dot and dashed load lines correspond to load impedances, respectively, smaller and higher than the optimum.

Assume that an input signal is applied driving the optimum load line between points $\mathrm{O} 1$ and $\mathrm{O} 2$. If the same input signal is applied within the other load cases, one can see that:

- for the higher load impedance, the swing across $v_{D S}$ is similar to that obtained with the optimum load, but the $i_{D S}$ excursion is limited to smaller values, leading to a drop in the output power.

- for the smaller load impedance, the $i_{D S}$ swing is equivalent to that of the optimum load, but the $v_{D S}$ excursion is smaller. The voltage gain increases, but the output power decreases.

These two cases show also that observing only either voltage or current does not reflect the actual power delivered to the load. That is also seen in Figs. 2 and 3 which show the corresponding $v_{d s}=\mathrm{f}\left(v_{g s}\right)$ and $i_{d s}=\mathrm{g}\left(v_{g s}\right)$ transfer characteristics for different loads.

The different behaviors of voltage and power are also seen in the sensitivities of the two quantities to load changes.

Figure 4 shows the variation of the output power with the load impedance obtained from the simulation of a class

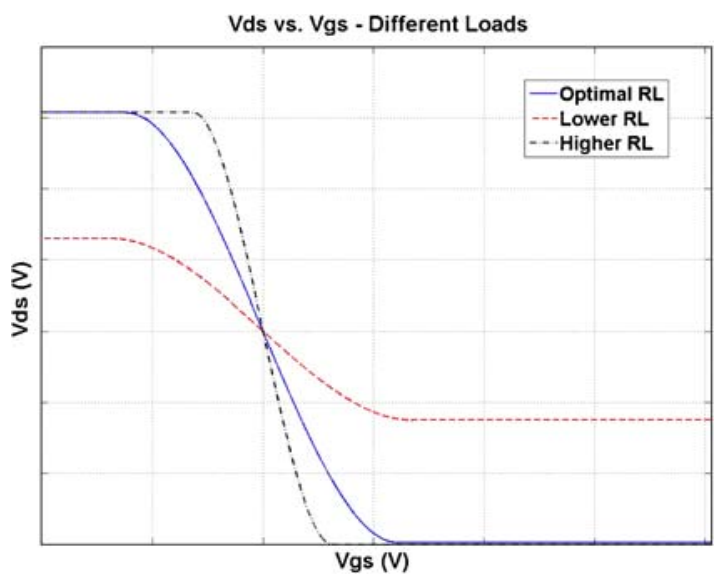

Fig. $2 v_{D S}$ vs. $v_{G S}$ excursions for different load resistance values

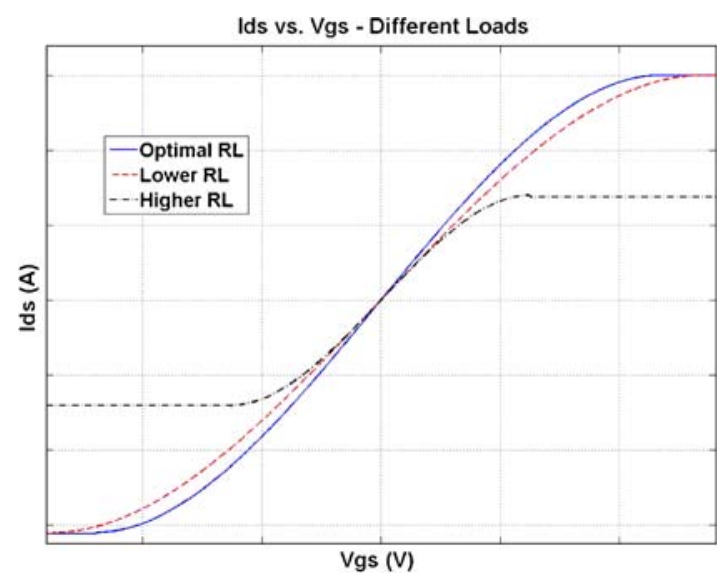

Fig. $3 i_{D S}$ vs. $v_{G S}$ excursions for different load resistance values

AB PA. The input signal was maintained constant and with the amplitude required to drive the PA into its maximum operating limits with the optimum load impedance. In these conditions, the load impedance was swept from half to the double of the optimum value and the corresponding load power and peak voltage were observed.

The fall-off of the power delivered to the load on either side of the maximum power transfer point $\left(\mathrm{Z}_{\mathrm{optimum}}\right)$ is the result of load VSWR (Voltage Standing-Wave Ratio) causing an ever increasing portion of the forward power to be reflected back to the amplifier.

Using classical transmission line theory, the real power delivered to the load can be found from $P_{L}=\left|\bar{a}_{L}\right|^{2}$ $\left(1-\left|\Gamma_{L}\right|^{2}\right)$, where $\left|\bar{a}_{L}\right|^{2}$ is the incident power at the load and $\Gamma_{L}=\left(Z-Z_{0}\right) /\left(Z+Z_{0}\right)$ the load voltage reflection coefficient that expresses the deviation of the load impedance from the characteristic (optimum) impedance $Z_{0}$ [3]. The slopes of the curve below and above this point, given by $d P_{L} / d \Gamma_{L}=-\left|\bar{a}_{L}\right|^{2}\left(2\left|\Gamma_{L}\right|\right)$, express the sensitivity of the load power to load variations.

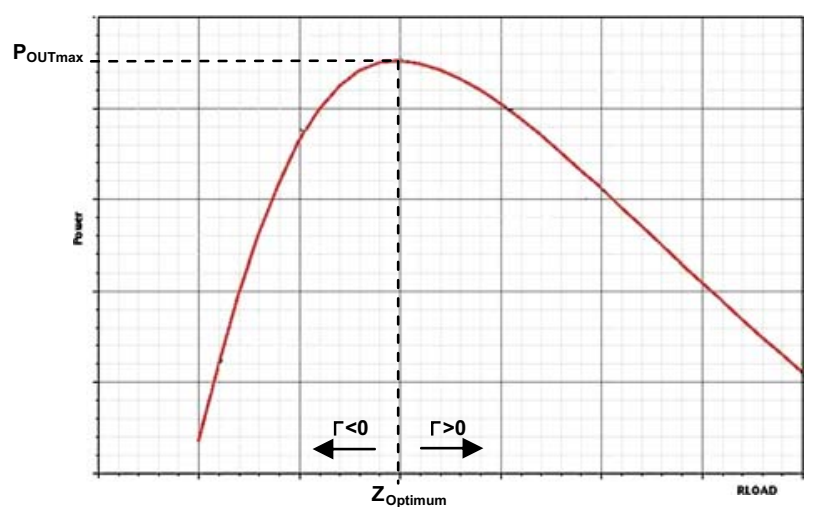

Fig. 4 Evolution of the power delivered to a varying load resistance 
The output voltage is given by $\overline{V_{L}}=\bar{a}_{L} \sqrt{Z_{0}}\left(1+\Gamma_{L}\right)$ and $d V_{L} / d \Gamma_{L}=\bar{a}_{L} \sqrt{Z_{0}}$.

Figures 5 and 6 show, respectively, the variation of the positive and negative output voltage peaks with the load resistance. From these two curves one can see that:

- the positive and negative excursions of the output voltage are different- - for the optimum load the response is symmetric but not for different load resistances

- the higher the load the higher the voltage excursion, but a large swing does not necessarily mean that the maximum power transmission has been achieved

- for load resistance values higher than the optimum the slope of the curve tends to decrease, as the transistor operating point reaches its limits, making it more difficult to detect load resistance variations

- it is not easily recognizable the point of maximum full power transmission.

These facts allow us to conclude that:

- the estimation of the linearity after peak voltage measurements requires that both positive and negative peaks are observed in order to obtain the worst case nonlinear characteristic.

- the observation of the actual output power does not allow either to know the symmetry of the transfer characteristic.

- observing the output power allows detecting the point of maximum power transmission. This fact can be explored to implement adaptive schemes capable of tuning the output matching network to ensure optimal operation.

- load resistance variations around the point of maximum power transfer are more easily detected observing the output voltage rather than output power, as the voltage presents a higher derivative at this point

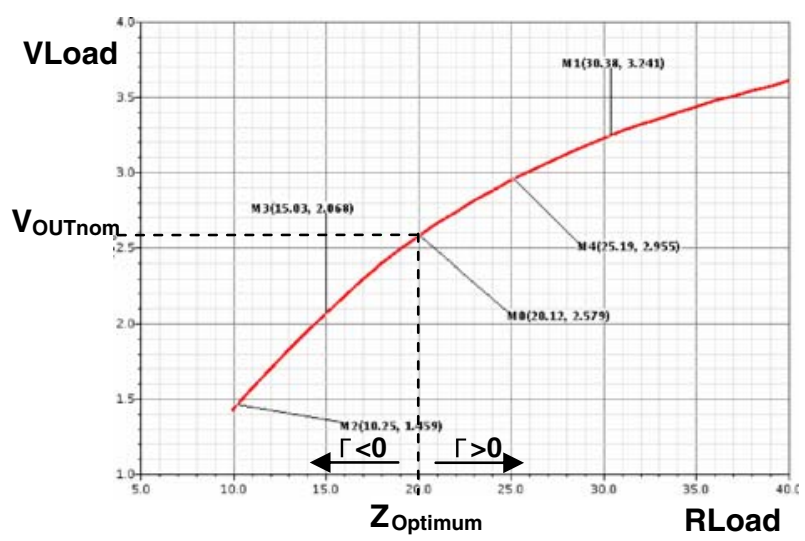

Fig. 5 Positive peak voltage for a varying load resistance

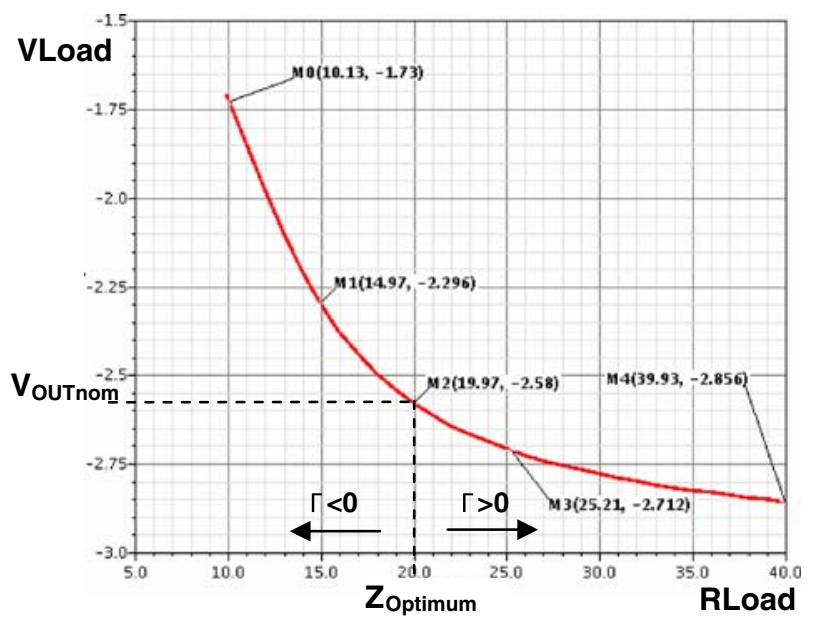

Fig. 6 Negative peak voltage for a varying load resistance

- as load resistance increases the voltage sensitivity to these variations decreases but not that of the output power $\left(d V_{L} / d Z=2 \bar{a}_{L} \sqrt[3]{Z_{0}} /\left(Z+Z_{0}\right)^{2}\right)$.

In RF measurements two types of sensors have been used: peak and root mean square (RMS) voltage sensors. It is commonly stated that the first ones give information on the peak power whereas the second ones give information on the average power. Often a peak detector is used to implement a protection to avoid transistor's breakdown due to overvoltage and an RMS detector is used to regulate PA's average output power for automatic level control and VSWR compensation.

In fact, in this context power and $V_{r m s}$ are used interchangeably [2] as power is commonly inferred from a measurement of root-mean-squared voltage taking an assumed known load resistance level, i. e. $P=V_{r m s}^{2} / R_{L}$, which is not totally correct as it was shown before. The methodology proposed here relies on obtaining the crosscorrelation between dynamic current and output voltage in order to perform an estimation of PA's nonlinearity based on actual power measurements.

\section{Estimation of Nonlinearity from Power Measurements}

It has been shown that a weakly nonlinear, memoryless, time-invariant, PA's voltage transfer characteristic can be expressed by a third-order polynomial [3]. This fact has been explored to develop in-circuit auxiliary test circuits based on the observation of different input and output RF voltage amplitudes $[1,11,15]$. Likewise, one can estimate a PA's power transfer characteristic after finding the polynomial $P_{\text {out }}=\beta_{1}$ Pin $+\beta_{2}$ Pin $^{2}+\beta_{3}$ Pin $^{3}$ that best fits a set of measured input-output power pairs, and whose coefficients allow one to compute $P i n_{1 d B}$ and $\operatorname{Pin}_{I I P 3}$ points. Following 
procedures similar to those presented in $[1,3,11]$ one can obtain, respectively,

$\left.\begin{array}{l}\operatorname{Pin}_{1 d B}=\frac{-\beta_{2}-\sqrt{\beta_{2}^{2}-4 \beta_{3}\left(\beta_{1}-\frac{\beta_{1}}{10^{\frac{1}{10}}}\right)}}{2 \beta_{3}} \\ \operatorname{Pin}_{I I P 3}=\sqrt{\left|\frac{\beta 1}{\beta 3}\right|}\end{array}\right\}$

to estimate $\operatorname{Pin}_{1 d B}$ and $\operatorname{Pin}_{I I P 3}$.
In order to obtain the three coefficients of the best fitting polynomial the following procedure was followed. If one obtains three output power levels from the PA under test $\left(\mathrm{P}_{\text {out } 1,2,3}\right.$ in Fig. 7$)$, the values of the three coefficients $\beta_{1}, \beta_{2}$, and $\beta_{3}$, can be obtained from the set of Eq. 3. which after applying Cramer's rule (2). provide (1). Nevertheless, the three testing stimuli amplitudes $P_{\text {in } 1,2,3}$ have to be optimized in order to obtain the best estimation accuracy for $\operatorname{Pin}_{1 d B}$ and Pin $_{I I P 3}$.

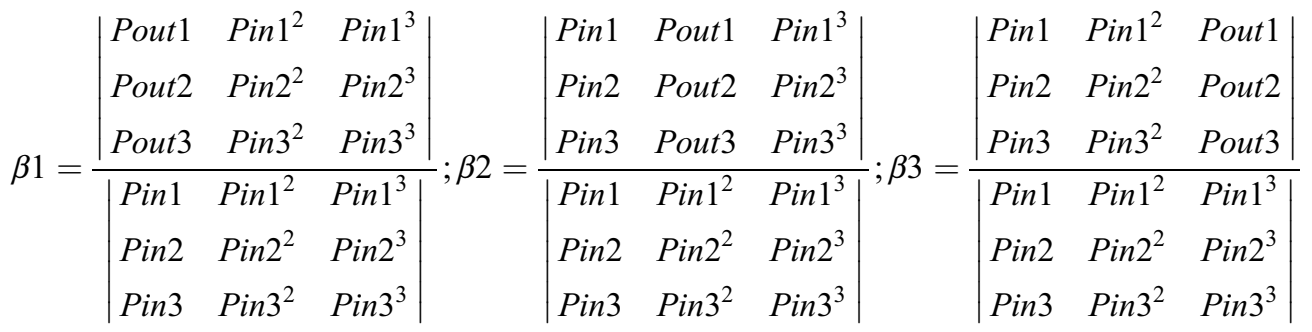

In order to find the best set of three stimuli to be used a minimum search function $\min f(x)$ was employed, where $x$ is the vector of the three stimuli amplitudes and $f(x)$ is the error occurred in the computation of $\operatorname{Pin}_{1 d B}$.

$$
\left.\begin{array}{l}
\text { Pout } 1=\beta 1 \text { Pin } 1+\beta 2 \text { Pin } 1^{2}+\beta 3 \text { Pin } 1^{3} \\
\text { Pout } 2=\beta 1 \text { Pin } 2+\beta 2 \text { Pin } 2^{2}+\beta 3 \text { Pin } 2^{3} \\
\text { Pout } 3=\beta 1 \text { Pin } 3+\beta 2 \text { Pin }^{2}+\beta 3 \text { Pin } 3^{3}
\end{array}\right\}
$$

Simulation results obtained within the Agilent ADS tool using a class AB PA, revealed that choosing Pin $_{1}$ and $\mathrm{Pin}_{3}$ in order to be equally distant from $\mathrm{Pin}_{2}$ and $P i n 1<\operatorname{Pin}_{1 d B}<P i n 3$, and $\operatorname{Pin}_{2}$ close to the expected $\operatorname{Pin}_{1 d B}$ (Fig. 7), a very accurate $\operatorname{Pin}_{1 d B}$ estimation can be obtained. Figure 8 allows one to compare the actual

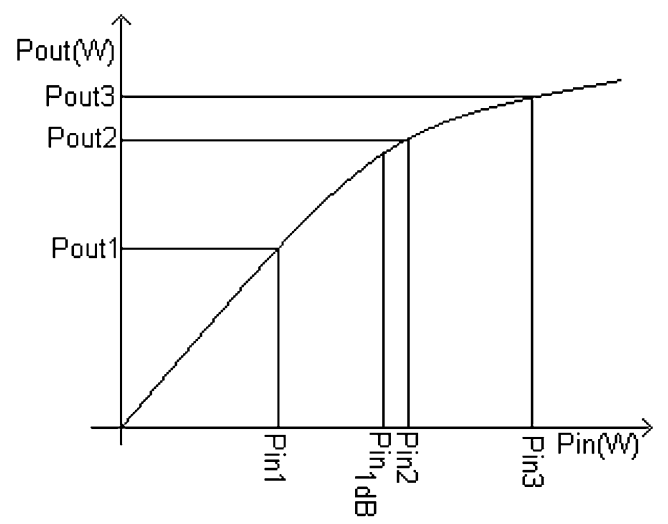

Fig. 7 Placement of the three test stimuli in a generic PA's transfer characteristic power transfer curve against the extrapolated one found with this procedure, and reveals that a very good match is obtained.

Figure 9 shows the variation of the $\operatorname{Pin}_{1 d B}$ estimation error with the placement of the three stimuli in relation to the expected $\operatorname{Pin}_{1 d B}$, keeping constant the intervals among them. It can be seen that when the intermediate value $\left(\operatorname{Pin}_{2}\right)$ of the three stimuli is close to the expected $P i n_{1 d B}$, the error is very small. In fact, very similar estimated and expected values were obtained for $\operatorname{Pin}_{1 d B}$, i. e.,

$\operatorname{Pin}_{1 \mathrm{dBest}} \approx \operatorname{Pin}_{1 \mathrm{~dB} \exp }=4,67 \mathrm{dBm}$

Placing the intermediate stimulus amplitude close to the expected $\operatorname{Pin}_{1 d B}$ point allows also for minimizing the deviation due to random noise in voltage and current signals.

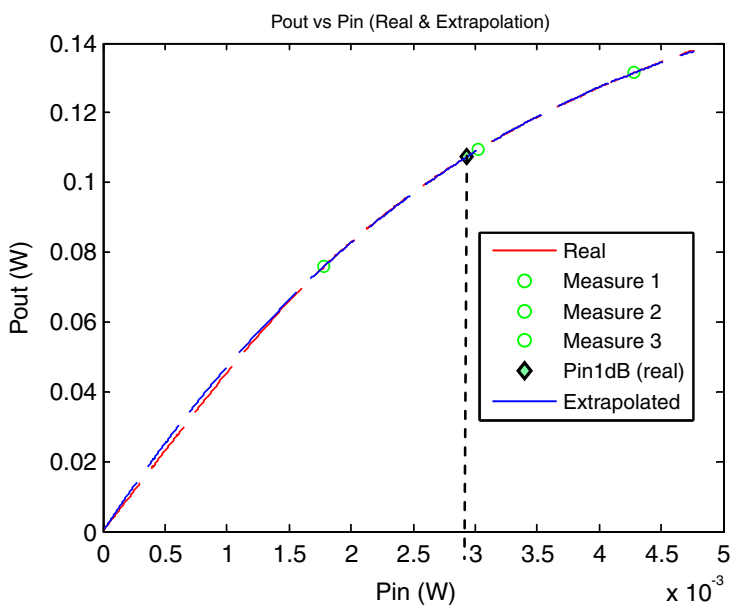

Fig. 8 Power transfer characteristics of a class $A B$ amplifier 


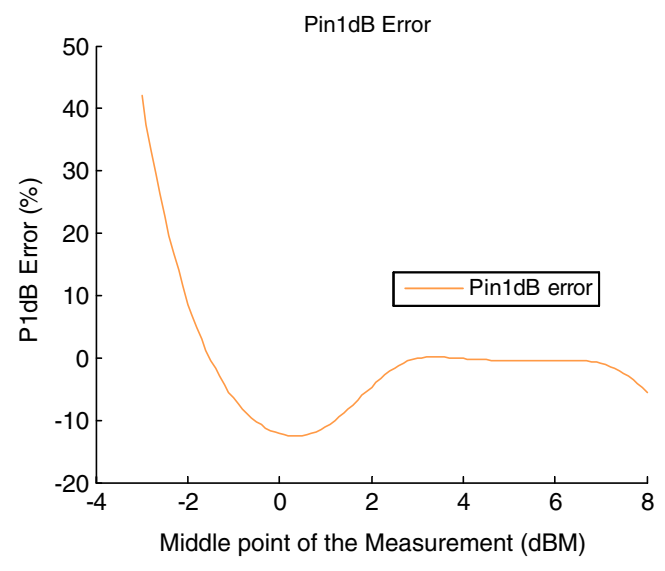

Fig. $9 \operatorname{Pin}_{1 d B}$ estimation error

Simulations superimposing random noise, with a $\pm 30 \mathrm{~dB}$ signal to noise ratio, were taken to observe this behavior. Also, different power transfer curves were tested.

Concerning the estimation of IP3 small errors can also be obtained but the sensitivity to the placement of the three stimuli amplitudes in the transfer characteristic is higher. Anyway, the obtained estimated value is Pin $_{I I P 3 e s t}=12.5 \mathrm{dBm}$ while the expected one is $\operatorname{Pin}_{I I P 3} \exp =13 \mathrm{dBm}$, i. e., an error of $-3.8 \%$ occurs.

A successive approximate procedure can be implemented using as new stimuli middle point the estimated $P i n_{1 d B}$ in case this is different from the expected one, until a convergence is obtained.

It is shown in $[1,11]$ that the estimation of IIP3 from PA's gain compression curves provides results with acceptable accuracy, but which may eventually require considering higher order fitting polynomials when amplifiers' nonlinearities cannot be considered weak. Further work should be carried-out on this matter.

\section{Cross-Correlation Based Power Measurement}

As it was shown before, looking at voltage amplitudes, does not provide a fully accurate power measurement in case an impedance deviation has occurred. This requires observing the actual power in the nodes.

The cross-correlation between two signals, $x(t)$ and $y(t)$, is defined as $\Re(\tau)=\int_{-\infty}^{+\infty} x(t)^{*} y(t+\tau) d t$, where $\tau$ is a time delay, which becomes ${ }^{-\infty}$

$\Re(\tau)=\frac{1}{2} X Y \cos (\omega \tau+\theta)$

when $x(t)$ and $y(t)$ are periodic signals of period $\mathrm{T}$, given by $x(t)=X \sin (\omega t)$ and $y(t)=Y \sin (\omega t+\theta)$. If $x(t)$ and $y(t)$ are the voltage and current of a circuit, (5) provides the circuit's active power when time delay $\tau$ is null. That is,

$$
\begin{aligned}
& \Re v i(0)=\frac{1}{2} V I \cos (\theta) \equiv \\
& P=\frac{1}{T} \int_{0}^{T} V(t) \times I(t) d t=\frac{1}{2}(V \times I) \times \cos (\theta)
\end{aligned}
$$

The correlation output provides a DC voltage proportional to the active power. In case voltage and current present different tones, the measurement provided by the correlator also includes the power due to intermodulation tones.

Consider that the amplifier output voltage and current can be represented by the polynomials (6) and (7), respectively.

$V(x)=a_{3} \cdot x^{3}+a_{2} \cdot x^{2}+a_{1} \cdot x$

$I(x)=b_{3} \cdot x^{3}+b_{2} \cdot x^{2}+b_{1} \cdot x$

If a two tone stimuli $x=A \cdot \cos \left(\omega_{1}\right)+A \cdot \cos \left(\omega_{2}\right)$ is applied to the amplifier then multiples of the fundamental frequencies, as well as intermodulation products, are generated due to the nonlinearity of the transfer characteristic. The output voltage and current are then given by

$$
\begin{aligned}
V(\omega)= & k_{1} \cdot\left(\cos \left(\omega_{1}\right)+\cos \left(\omega_{2}\right)\right)+k_{2} \cdot\left(\cos \left(2 \cdot \omega_{1}\right)\right) \\
& +\cos \left(2 \cdot \omega_{2}\right)+\cdots k_{3} \cdot\left(\cos \left(3 \cdot \omega_{1}\right)+\cos \left(3 \cdot \omega_{2}\right)\right)+ \\
& +k_{4} \cdot\left(\cos \left(\omega_{1}-\omega_{2}\right)+\cos \left(\omega_{1}+\omega_{2}\right)\right)+\cdots \cdots \\
& +k_{5} \cdot\left(\begin{array}{l}
\cos \left(2 \cdot \omega_{1}-\omega_{2}\right)+\cos \left(2 \cdot \omega_{1}+\omega_{2}\right) \\
+\cos \left(2 \cdot \omega_{2}-\omega_{1}\right)++\cos \left(2 \cdot \omega_{2}+\omega_{1}\right)
\end{array}\right)
\end{aligned}
$$

$$
\begin{aligned}
I(\omega)= & m_{1} \cdot\left(\cos \left(\omega_{1}\right)+\cos \left(\omega_{2}\right)\right) \\
& +m_{2} \cdot\left(\cos \left(2 \cdot \omega_{1}\right)+\cos \left(2 \cdot \omega_{2}\right)\right) \\
& +\cdots m_{3} \cdot\left(\cos \left(3 \cdot \omega_{1}\right)+\cos \left(3 \cdot \omega_{2}\right)\right)+ \\
& +m_{4} \cdot\left(\cos \left(\omega_{1}-\omega_{2}\right)+\cos \left(\omega_{1}+\omega_{2}\right)\right)+\cdots \cdots \\
& +m_{5} \cdot\left(\begin{array}{c}
\cos \left(2 \cdot \omega_{1}-\omega_{2}\right)+\cos \left(2 \cdot \omega_{1}+\omega_{2}\right) \\
+\cos \left(2 \cdot \omega_{2}-\omega_{1}\right)+\cos \left(2 \cdot \omega_{2}+\omega_{1}\right)
\end{array}\right)
\end{aligned}
$$

where coefficients $k_{i}$ and $m_{i}$ depend of amplitude A and coefficients $a_{i}$ and $b_{i}$, respectively.

After the multiplication of voltage and current signals, the DC terms, i. e., average power, returns also the 
contribution of the power of other tone components besides the fundamentals, as

$P_{a v g}=k_{1} \cdot m_{1}+k_{2} \cdot m_{2}+k_{3} \cdot m_{3}+k_{4} \cdot m_{4}+2 \cdot k_{5} \cdot m_{5}$

That would not be the case with voltage detectors.

\section{The Cross-Correlator Circuit}

The correlation circuit shown in Fig. 10, implemented with a mixer and a low-pass filter, was used to evaluate the applicability of cross-correlating current and output voltage to estimate $\operatorname{Pin}_{1 d B}$ and $\operatorname{Pin}_{I I P 3}$. The voltage and current signals are filtered in order that only the respective fundamental components, $v_{o u t_{1}}$ and $i_{d d_{1}}$, are observed.

The correlator's AC output voltage is obtained from the voltage developed at the output of the differential pair, after the product of $i_{S}$ by the small signal output resistance seen in the drain of $\mathrm{M}_{3} . i_{S}$ results from the difference between currents $i_{1}$ and $i_{2}$.

On the other hand, $v_{\mathrm{x}}$ is given by the product of transistor's M1 transconductance current, which depends on $v_{\text {out }}$, by the output resistance of transistor $\mathrm{M}_{1}$. The correlator's input differential voltage $v_{d}$ (which is proportional to the PA's output current) is obtained from current $i_{d d 1}$ and the impedance of the network used to filter the dynamic signal current (Fig. 11).

The derivation of the correlator's output current $i_{S}$ is shown in Eq. 11, where, the differential voltage at the correlator's input terminals, $v d$, is split in $v d / 2$ and $-v d / 2$, and then replaced in the equations of transistors' currents in saturation mode. After developing the square and eliminating terms with opposite sign, one gets as final result the

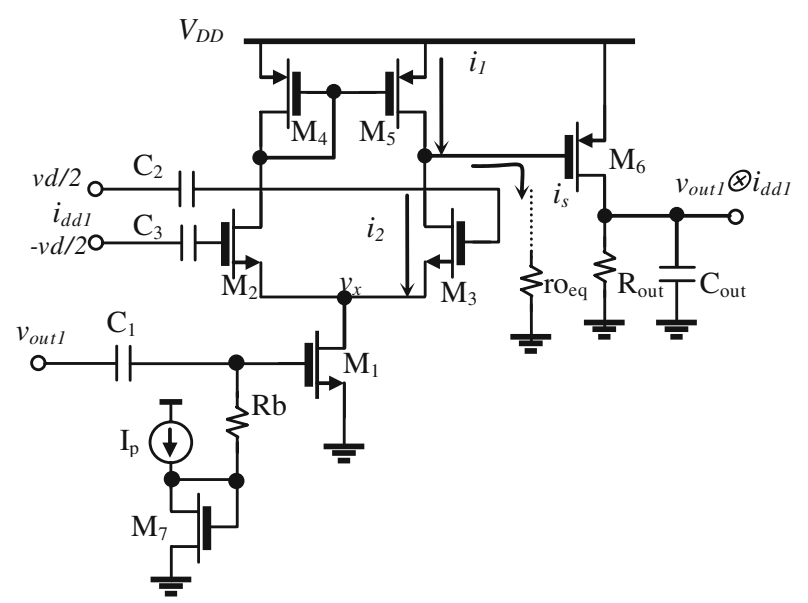

Fig. 10 Correlator circuit

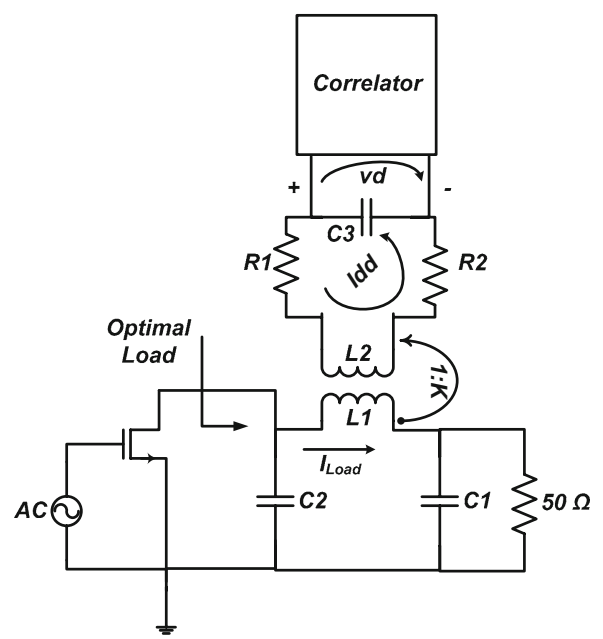

Fig. 11 Sensing circuit for the correlator current input

product between the differential voltage $v d$ and the PA's output voltage.

$$
\begin{aligned}
& i_{S}=i_{1}-i_{2} \quad \theta=V_{B I A S}-V_{X}-V_{t h} \quad W=W_{2}=W_{3} \\
& K=K_{2}=K_{3} \quad V_{B I A S}=V_{G 2}-V_{G 3} \\
& i_{s}=\frac{k}{2} \frac{W}{L} \cdot\left(\frac{v d}{2}-v_{x}+\theta\right)^{2}-\frac{k}{2} \frac{W}{L} \cdot\left(-\frac{v d}{2}-v_{x}+\theta\right)^{2} \\
& i s=\frac{k}{2} \frac{W}{L} \cdot\left(\left(\frac{v d}{2}\right)^{2}-v d \cdot v_{x}+v_{x}^{2}-2 \cdot\left(\frac{v d}{2}-v_{x}\right) \cdot \theta+\theta^{2}\right)+\ldots \\
& \ldots-\frac{k}{2} \frac{W}{L} \cdot\left(\left(\frac{v d}{2}\right)^{2}+v d \cdot v_{x}+v_{x}^{2}-2 \cdot\left(-\frac{v d}{2}-v_{x}\right) \cdot \theta+\theta^{2}\right) \\
& i s=\frac{k}{2} \frac{W}{L}\left(\begin{array}{l}
-v d \cdot v_{x}-v d \cdot v_{x}-2 \cdot(\frac{v d}{2}+\frac{v d}{2}+\overbrace{v_{x}-v_{x}}^{0}) \cdot \theta+\ldots \\
\ldots+\underbrace{\theta^{2}-\theta^{2}}_{0}+\overbrace{\left(\frac{v d}{2}\right)^{2}-\left(\frac{v d}{2}\right)^{2}}^{0}+\overbrace{v_{x}^{2}-v_{x}^{2}}^{0}
\end{array}\right) \\
& i s=\frac{k}{2} \frac{W}{L}\left(-2 \cdot v d \cdot V_{x}-v d \cdot \theta\right) \\
& i_{s}=\frac{k}{2} \frac{W}{L}(2 \cdot g m_{1} \cdot r o_{1} \cdot \underbrace{v d \cdot v_{\text {out }}}_{\text {Product }}-\underbrace{v d \cdot \theta}_{\text {Linear }})
\end{aligned}
$$

Equation 11 shows that $i_{S}$ is proportional to the product between $v_{d}$ (proportional to $i_{d d}$ ) and PA's $v_{\text {out }}$ and thus provides a measure of the PA's output power. The final output voltage is achieved after a final gain stage which provides an output voltage given by, before filtering,

$V_{\text {corr }}=\frac{k}{2} \frac{W}{L} \cdot(2 \cdot g m_{1} \cdot r o_{1} \cdot \underbrace{v d \cdot v_{\text {out }}}_{\text {Pr oduct }}-\underbrace{v d \cdot \theta}_{\text {Linear }}) \cdot g m_{6} \cdot r o_{6}$

After filtering the $2 \omega$ term due to the $v d . v_{\text {out }}$ product and the $\omega$ term due to the $v d . \theta$, one will have a DC voltage which represents the active power. 


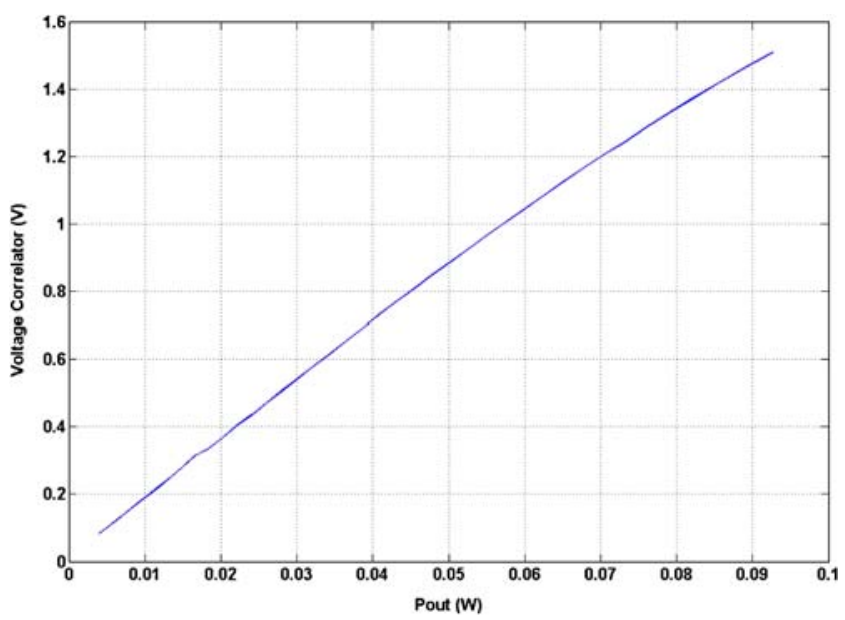

Fig. 12 Correlator output voltage vs. PA's output power

The current used in the correlation is a scaled sample of that flowing into the load. To perform this, a transformer is included in the circuit (Fig. 11), where the coupling factor determines the transfer ratio among the two currents. The two inductors that form this transformer play different roles in the circuit. It provides both impedance matching as well as output current sensing. Resistors R1 and R2 connected to $\mathrm{L} 2$ are included to accommodate the input signal $v_{d}$ to the correlator's dynamic range when operating in linear mode.

Figure 12 shows that a good linearity exists between the correlator's output voltage and the power to be measured. That is also seen in Fig. 13, which shows both the PA's actual transfer characteristic $\left(\mathrm{P}_{\text {out }}\right.$ real) and that observed with the correlation based sensor. The good matching between the two curves allows us to estimate $\operatorname{Pin}_{1 d B}$ and

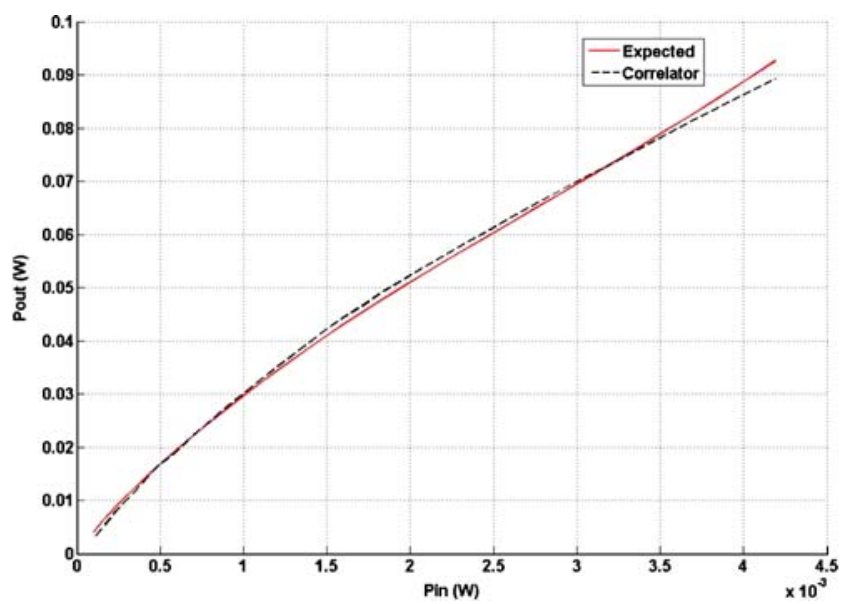

Fig. 13 Comparison between the actual power transfer curve and that obtained with the correlator sensor

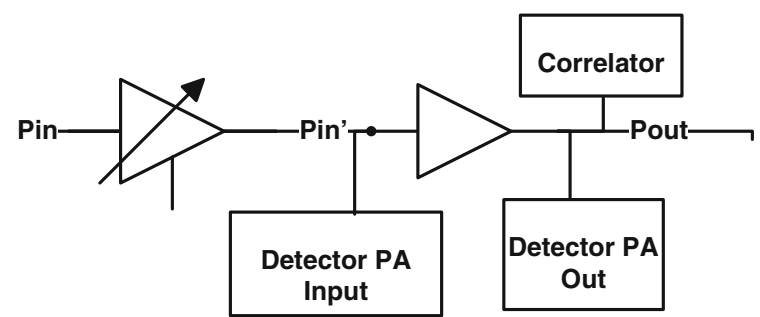

Fig. 14 Block diagram of prototype chip

$\operatorname{Pin}_{I I P 3}$ with the same accuracy as that obtained using voltage and current output values, i.e.,

Pin $_{1 \mathrm{dBest}}=4,67 \mathrm{dBm}$ and Pin $_{\text {IIP3est }}=12.5 \mathrm{dBm}$.

as presented before.

\section{Demonstration Prototype}

A demonstration prototype chip was designed within a CMOS $0.35 \mu \mathrm{m}$ technology and sent for fabrication. It implements a power amplifier, a pre-amplifier and the correlator, as well as peak voltage detectors (Fig. 14). The pre-amplifier is a digitally controlled variable gain amplifier usually employed to control PA's output power, but that in this case can also be used for testing purposes to generate the different amplitude stimuli.

Using this circuit simulations were carried-out to compare the accuracy of the measures obtained when using the voltage detectors to those obtained with the correlator.

First, the voltage detectors were calibrated to provide a linear response to the output peak voltage for the optimum load case. It can be seen in Fig. 15 that good linearity is ensured and thus that the transfer characteristics obtained

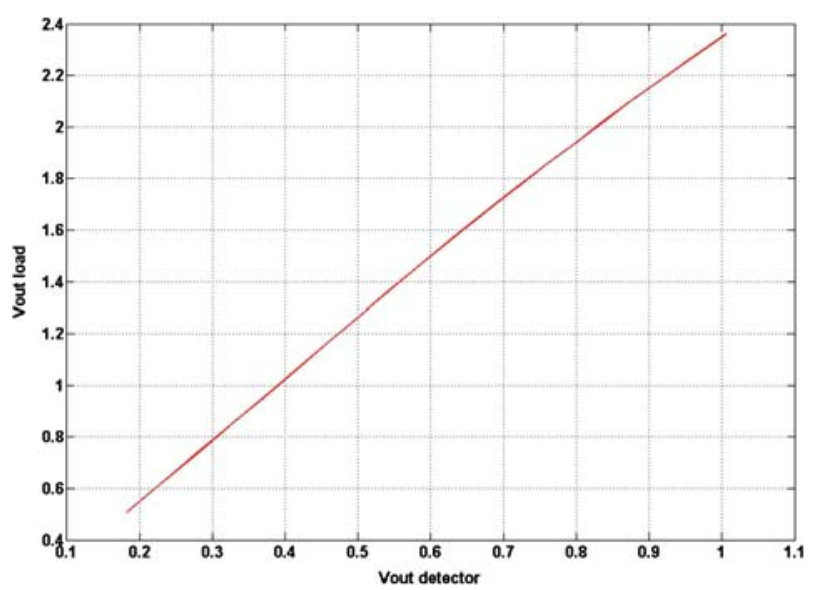

Fig. 15 Voltage provided by the output peak detector as a function of load power 


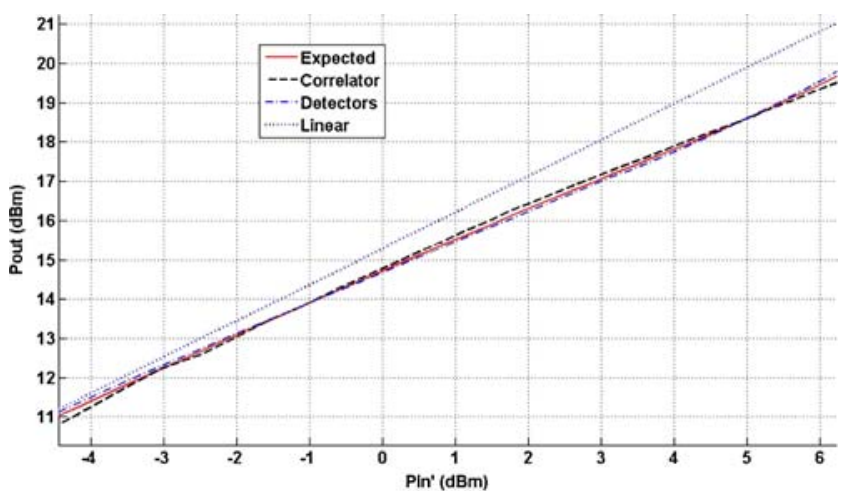

Fig. 16 Pout vs Pin' transfer characteristics obtained with the peak detectors and the correlator for nominal load

with both the correlator and voltage detectors are not affected by their respective behaviors.

Using the values given by the voltage detectors and the correlator, the power transfer characteristics for different load resistances were obtained. Figure 16 shows the PA's Pout vs. Pin' (Fig. 14) transfer characteristics obtained with the correlator and the voltage detectors, together with the expected one, for the nominal load. It can be seen that, in this situation, a good matching among the three curves is obtained along the entire operating range.

However, if load impedance changes, the power measured with the peak voltage detectors is different as their response has been calibrated for the nominal optimum load resistance (Fig. 17), i. e., power $\left(P=V_{p k}^{2} / 2 R_{L}\right)$ is computed using the expected load resistance value and not the actual ones, rending thus, respectively, smaller and higher values than expected. The measurements provided by the correlator are more accurate than those obtained with the peak voltage detectors. Only for low capacitive impedances the detector provides a better accuracy.

Figure 18 shows the Pout vs Pin' transfer characteristics obtained for load resistances a) smaller $(-60 \%)$ and b) higher $(40 \%)$ than the optimum load resistance. Although the measures provided by the correlator differ slightly from the expected ones still provide a better estimation than that given by the peak voltage detector. This difference is now due to the correlator $\left(v_{d}\right.$ and $v_{\text {out }}$ inputs, Fig. 10) being operated far from the nominal operating point.

Figure 19 shows the layout of the correlator. It has an area of $80 \times 80 \mu \mathrm{m}^{2}$, mostly occupied by the capacitors and bias resistors. Nevertheless, this area is less than $10 \%$ the PA area (inductors included) representing thus a small area
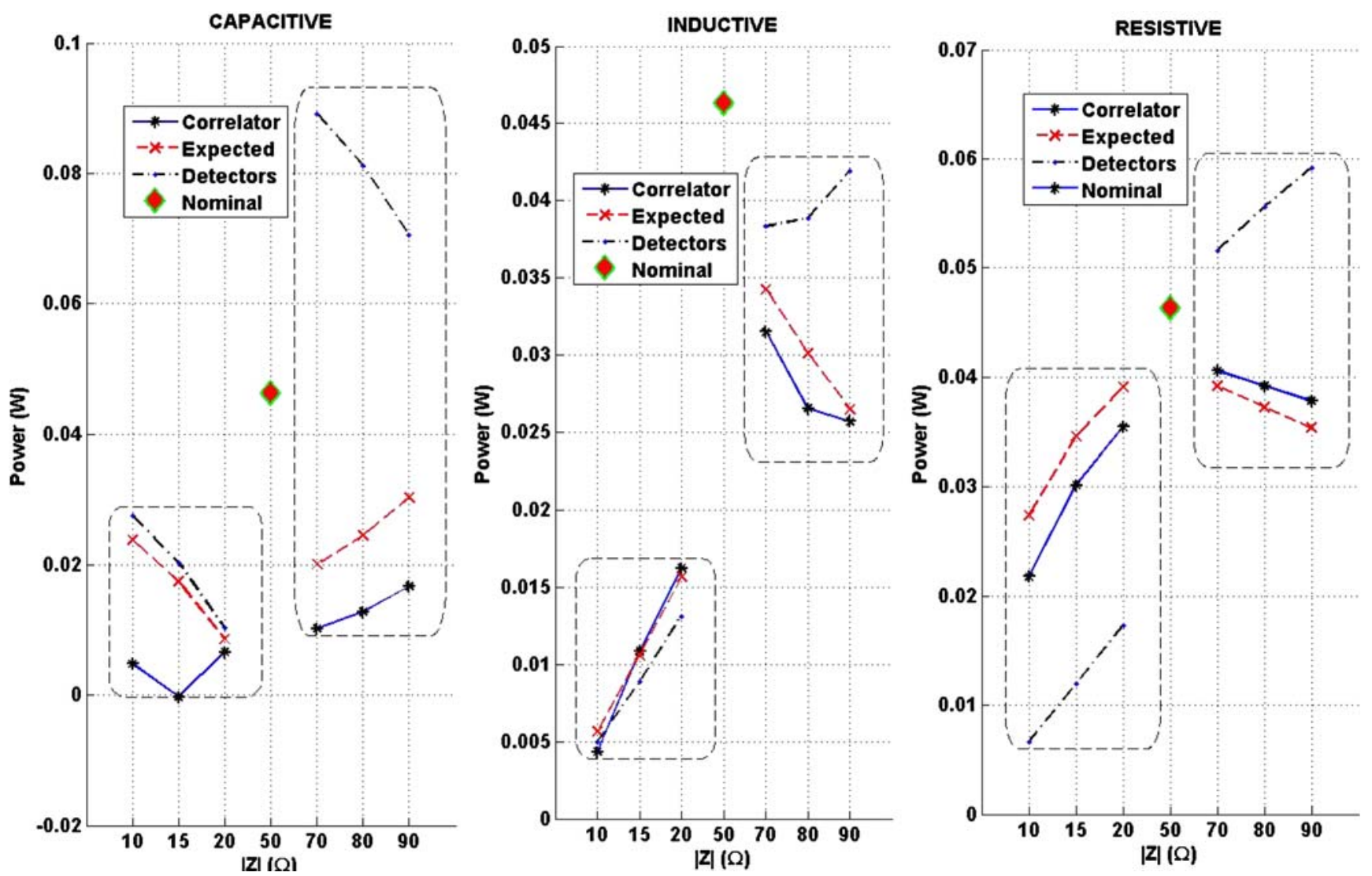

Fig. 17 Output power measured for different load impedances 


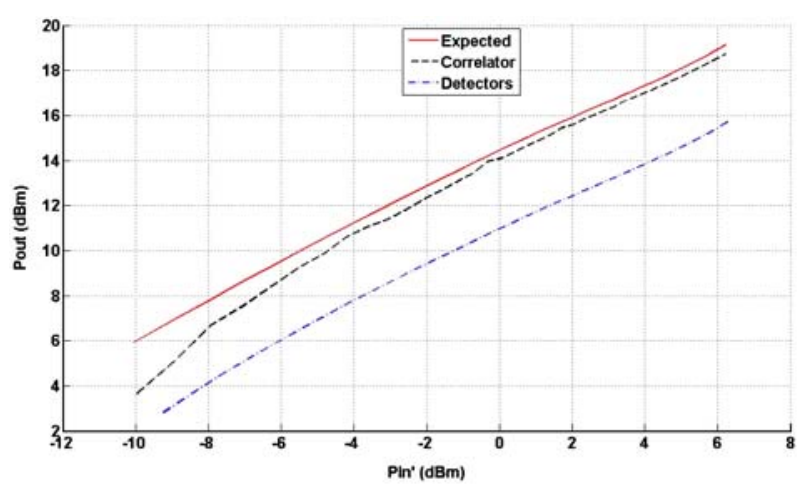

a)

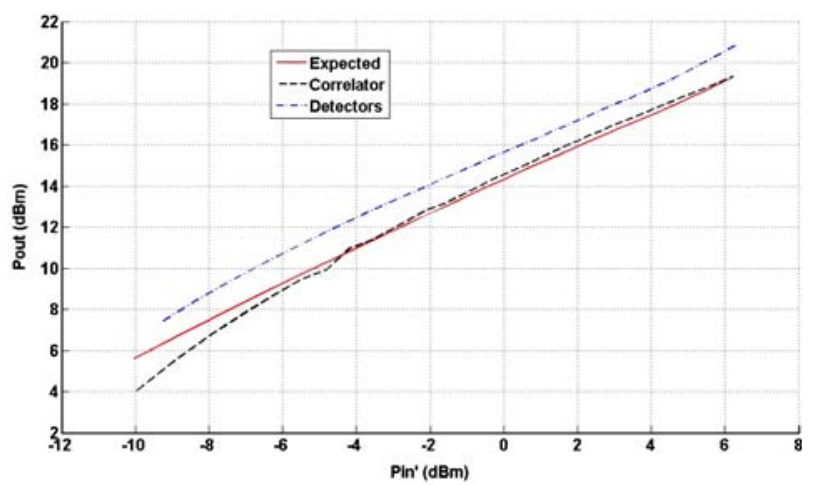

b)

Fig. 18 Pout vs Pin' transfer characteristics obtained for load resistances a) $60 \%$ reduction and b) $40 \%$ increase of load resistance

overhead. At the time of writing the fabricated chips had not yet been received.

The testing scheme being described here only provides the means to generate (three) stimuli amplitudes and detect the corresponding output power levels. These can be measured as DC voltages, and thus a general purpose test bus can be used.

The computation of $\operatorname{Pin}_{1 d B}$ and $\operatorname{Pin}_{I I P 3}$ values has to be performed off-chip using the set of power pairs observed in response to the three test stimuli. However, since a complete transceiver also includes a DSP unit, one can use these resources to implement the mathematical operations needed to calculate the coefficients and thus provide a full built-in testing operation.

\section{Conclusion}

The development of built-in self-test solutions for radio frequency circuits has not yet reached the maturity required to allow replacing comprehensive, but time-consuming characterization testing operations, for tests capable to screen malfunctioning circuits due to processing defects and process variations.
The work presented herein addresses the estimation of RF power amplifiers' $1 \mathrm{~dB}$ compression and third-order intercept $\left(\mathrm{P}_{1 \mathrm{~dB}}\right.$ and IP3) nonlinearity figures obtained after cross-correlating dynamic output voltage and current. This allows obtaining a measure of the actual output power rather than estimations based in voltage measurements, as it is usually done.

It is shown here that load impedance variations are not equally detected when observing voltage and when observing the actual power. Results obtained with a crosscorrelator implemented with a simple Gilbert mixer and low-pass filter, show that good power measurement accuracy can be obtained which allow also estimating $\mathrm{P}_{1 \mathrm{~dB}}$ and IP3 with small errors. The polynomial fitting approach presented here allows obtaining these parameters with just three stimuli levels. These estimations are more accurate than those provided by voltage measurements (either peak or RMS) in case load impedance variations occur.

Eventually voltage and actual power detectors could be used together. Relying on actual power measurements one can implement regulation loops for automatic level control (ALC) namely to improve linearity, and correct the PA operation for process, voltage and temperature variations. Also, by detecting load impedance variations, a regulation loop can limit the voltage standing wave ratio to improve linearity and power transmission. The voltage detectors

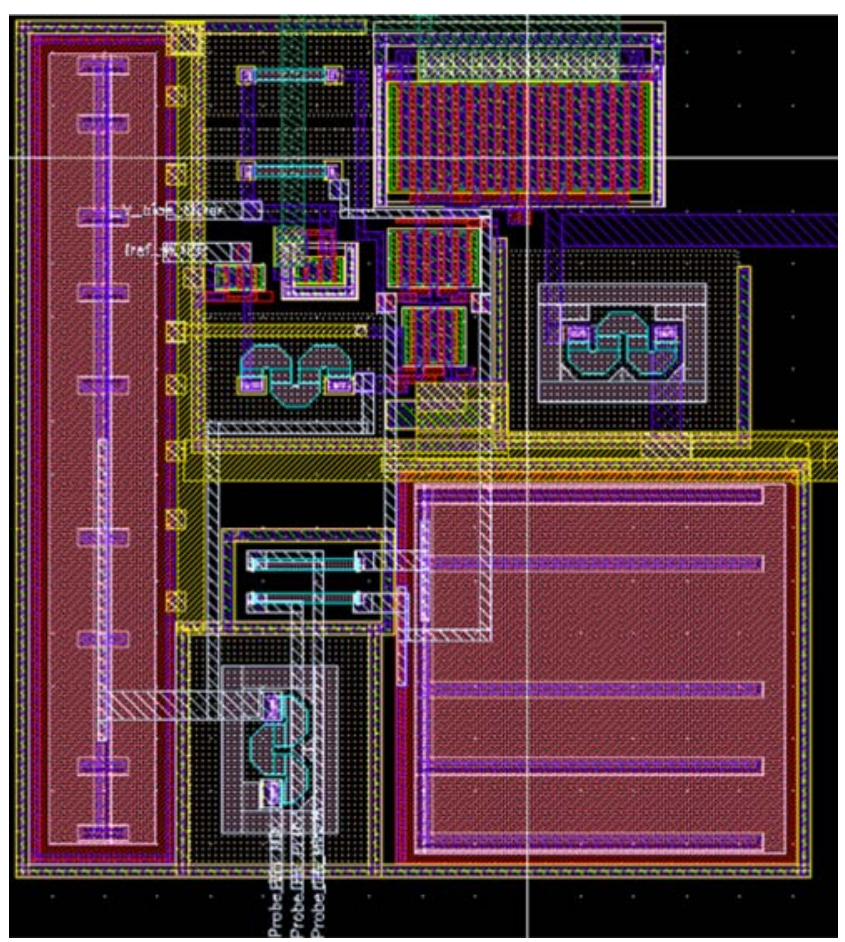

Fig. 19 Layout of the correlator 
allow detecting asymmetries in the output voltage and overvoltages allowing thus for protecting the power amplifier against the occurrence of operating points in the breakdown region.

\section{References}

1. Choongeol C, Eisenstadt WR, Stengel B, Ferrer E (2005) IIP3 Estimation from the gain compression curve. IEEE Transactions On Microwave Theory and Techniques. 53(4)

2. Cowles JC (2004) The evolution of integrated RF power measurement and control. IEEE MELECON 2004. Dubrovnik, Croatia

3. Ellinger F (2007) Radio frequency integrated circuits and technologies. Springer-Verlag, Berlin

4. Ellouz S, Gamand P, Kelma C, Vandewiele B, Allard B (2006) Combining internal probing with artificial neural networks for optimal RFIC testing. IEEE international test conference. pp. 1-9

5. Fan X, Onabajo M, Fernandez-Rodriguez FO, Silva-Martinez J, Sanchez-Sinêncio E (2008) A current injection built-in test technique for RF low-noise amplifiers. IEEE Transactions on Circuits and Systems I 55(7):1794-1804

6. Ferrario J, Wolf R, Moss S, Slamani M (2003) A low-cost test solution for wireless phone RFICs. IEEE communications magazine

7. Guillot F, Garcia P, Mouis M, Belot D (2006) Analysis of the intermodulation distortion and nonlinearity of common-base SiGeC HBTs. 13th IEEE International conference electronics, circuits and systems, ICECS '06. pp 664-667

8. Hafed M, Abaskharoun N, G. Roberts (2000) A stand-alone integrated test core for time and frequency domain measurements. Proceedings of the International Test Conference

9. Hassun R, Kuhm N, Posner R, Sweeney R, Vassilakis B (2001) Ultra-linear power amplifier characterization using dynamic range extension techniques. IEEE Symposium MTT

10. Maas SA, Microwave N, Circuits RF (2003) Nonlinear microwave and RF circuits. Artech House, Norwood
11. Mota P, da Silva JM, Long J (2007) Estimation and adaptive correction of PA's nonlinearities. 13th International mixed signals testing workshop and 3rd GHz/Gbps test workshop

12. Ryu J-Y, Kim BC, Sylla I (2006) A new low-cost RF built-in selftest measurement for system-on-chip transceivers. IEEE Transactions on Instrumentation and Measurement 55(2):381-388

13. Sokal NO, Sokal AD (1997) Accurate measurement of RF power amplifier efficiency and power output without and RF power meter. IEEE Journal of Solid-State Circuits. Vol. SC-12, No. 5

14. Strid, EW (2001) High-throughput RFIC wafer testing. ARFTG conference digest-spring, 57th Vol. 39:pp 1-5

15. Valdes-Garcia A, Silva-Martinez J, Sanchez-Sinêncio E (2006) On-chip testing techniques for RF wireless transceivers. IEEE Design \& Test of Computers 23(4):268-277

16. Voorakaranam R, Akbay SS, Bhattacharya S, Cherubal S, Chatterjee A (2007) Signature testing of analog and RF circuits: algorithms and methodology. IEEE Transactions on Circuits and Systems I 54(5):1018-1031

Pedro F. Mota obtained his five year degree in Electrical and Computer Engineering from the Faculty of Engineering of the University of Porto, Portugal, in 2005. He joined INESC Porto in 2005 as an assistant researcher and is currently concluding his $\mathrm{PhD}$ thesis on in-circuit testing of adaptive RF receivers.

José Machado da Silva graduated and obtained his $\mathrm{PhD}$, both in Electrical and Computer Engineering from the Faculty of Engineering of the University of Porto, Portugal, in 1984 and 1998, respectively.

He joined the University of Porto in 1984 as a lecturer, and INESC Porto in 1991 as a researcher. He is currently Associate Professor at UPorto and Project Leader at INESC Porto, with teaching and research responsibilities in design and test of electronic circuits and systems. His research interests include analog, mixed-signal, and RF circuits, MEMS and sensors, wireless networks, and analog and digital signal processing.

Ricardo A. Veiga obtained the MSc degree in Electrical and Computer Engineering from the Faculty of Engineering of the University of Porto, Portugal, in 2009. His MSc thesis was devoted to the evaluation of radio frequency power amplifiers non-linearities after the cross-correlation between dynamic output current and voltage. 